\title{
First-principles study of organic molecule for corrosion inhibition
}

\author{
Rachid Belkada ${ }^{1, *}$, Dalila Hammoutène ${ }^{2}$, Rahma Tibigui $^{2}$, and Ikram Hadj-Said $^{2}$ \\ ${ }^{1}$ CRTSE, Research Center in semi-conductor Technology for the Energetic, Algiers, Algeria \\ ${ }^{2}$ USTHB, Laboratory of Thermodynamics and Molecular Modelization, Faculty of \\ Chemistry, BP32 El Alia, 16111, Bab Ezzouar, Algiers, Algeria
}

\begin{abstract}
The widespread use of steel in various industries, especially in the transportation of hydrocarbons and gas, has recently gained a potential interest to explore eco-friendly solutions against corrosion. In fact, the highly aggressive environment generates considerable losses that affect global economy of countries that are mainly depending on the production and transport of energy. In the field of corrosion inhibitors, most common method so far available for protection against corrosion relies on synthetic one. These are unfortunately harmful to the environment as well to the human health, however they remain the most popular and the most effective due to their cost, and their ease of application. One of the most challenging issues in this area is the accurate understanding and measure of the degree of the passivation of corrosion inhibitors, which is complex and depend on many factors such as the nature of the metal, the fluid, the electronic structure of the inhibitor, the temperature, the exposure time, and so on. Recently, organic inhibitors have become increasingly attractive due to their competitive character as compared to the synthetic ones. With the use of advanced computational techniques enhanced by the development of density functional theory (DFT), it becomes possible to identify and design at the fundamental level, novel corrosion inhibitor molecules as complementary well established tool beside to the experimental techniques, which are often very expensive and timeconsuming. In this work, we explore by mean of DFT, the anti-corrosion effect of the Lawsone molecule (2-hydroxy-1,4-naphthoquinone) and some of its derivatives to clarify and understand the relationship at the fundamental level between the anti-corrosion properties and the structure of the molecule in contact with the iron.
\end{abstract}

\section{Introduction}

Nowadays, first-principles based fundamental calculations have proved its powerful position as a valid scientific tool to be integrated in the development of new technologies, especially in the field of material sciences and for innovative industrial solutions. This new

\footnotetext{
*Corresponding author: rbelkada@crtse.dz
} 
tool combined with the recent technological progress, which has been made in the field of organic material synthesis have led to the emergence of the novel organic compound used in various area with a wide applications, previously not accessible to technologies based experimental techniques $[1,2]$. As an example, organic materials have recently been well acknowledged in the field of corrosion protection due to their environmental friendly added values. The prediction of physical and chemical phenomena responsible for corrosion requires advanced numerical tools to deal with coupled interactions involving atoms and electrons, heat and species transport, which necessitates advanced quantum calculation techniques for an in-depth understanding of inherent phenomena. Although, corrosion inhibition and the electronic properties variation of an organic compound greatly affect the resistance to corrosion in contact with a metallic surface, it is likely dominated by energy difference near the band gap between the LUMO and HOMO. In fact, it has been shown that this energy difference is correlated to inhibition efficiency; the less the value of the energy difference, the higher impact on the inhibition efficiency. In such complex systems where interactions at fundamental level are involved, experimental investigations alone show its limitation and lack to consistently describe the correlation between the energy variation and capacity of efficient inhibition. The fundamental description of theses phenomena, in particular the electron transfer from the inhibitor to the metallic atom of the protected surface can be undertaken with a good accuracy using the density functional theory (DFT) [3-5]. The present work consists of ab initio based fundamental calculations of ten di-chlorinated Lawsone derivatives in the neutral, cationic and anionic states. This study aims for understanding of relationship between the passivation properties of an organic molecule considering its different derivatives in the presence with an iron atom. The calculated electronic properties clearly indicated the correlation between the transfer of charges and the inhibition character of the Lawsone molecule.

\section{Calculation method}

The geometries of (2-hydroxy-1,4-naphthoquinone) and calculations on all ten dichloridederivatives as shown in Table 1, have been performed by density functional theory (DFT), as implemented in the Gaussian 09 quantum chemistry code [6], using the hybrid functional B3LYP combined with the $6-311 \mathrm{G}^{* *}$ basis set. For all these systems, we have completely optimized the different geometries in the neutral, cationic and anionic states. The results obtained allowed us to obtain the total energy Etot, the ionization potential I and the electronic affinity $\mathrm{A}$, which will be used to calculate the transferred electron fraction $\Delta \mathrm{N}$, giving us information about the corrosion inhibition property of our different systems. As a measure of the passivation potential of the organic molecule inhibitor on the fraction of the electron transfer $(\Delta \mathrm{N})$ from inhibitor to the iron atom, this is expected at its large value to exhibit a high vulnerability to corrosion. The fraction of electrons transferred from the inhibitor to the iron atom is given by:

$$
\Delta \mathrm{N}=\frac{\left(\chi_{F e}-\chi_{i n h}\right)}{2\left(\eta_{F e}-\eta_{i n h}\right)}
$$

where a theoretical electronegativity value of $\chi_{F e}=7.9024 \mathrm{eV}$ is taken for the iron and having the absolute hardness of $\eta_{F e}=0$, where in our case the ionization potential, I and the electron affinity, $\mathrm{A}$ are equal $(\mathrm{I}=\mathrm{A})$ at the metal bulk. Indeed, the difference between the energy levels HOMO and LUMO in the molecule of the inhibitor is another important parameter. In fact the low values of the gap energy (Egap = ELUMO - EHOMO) are a sign of good inhibitory efficiency. 
Table 1. List of the investigated di-chlorinated Lawsone derivatives molecules.

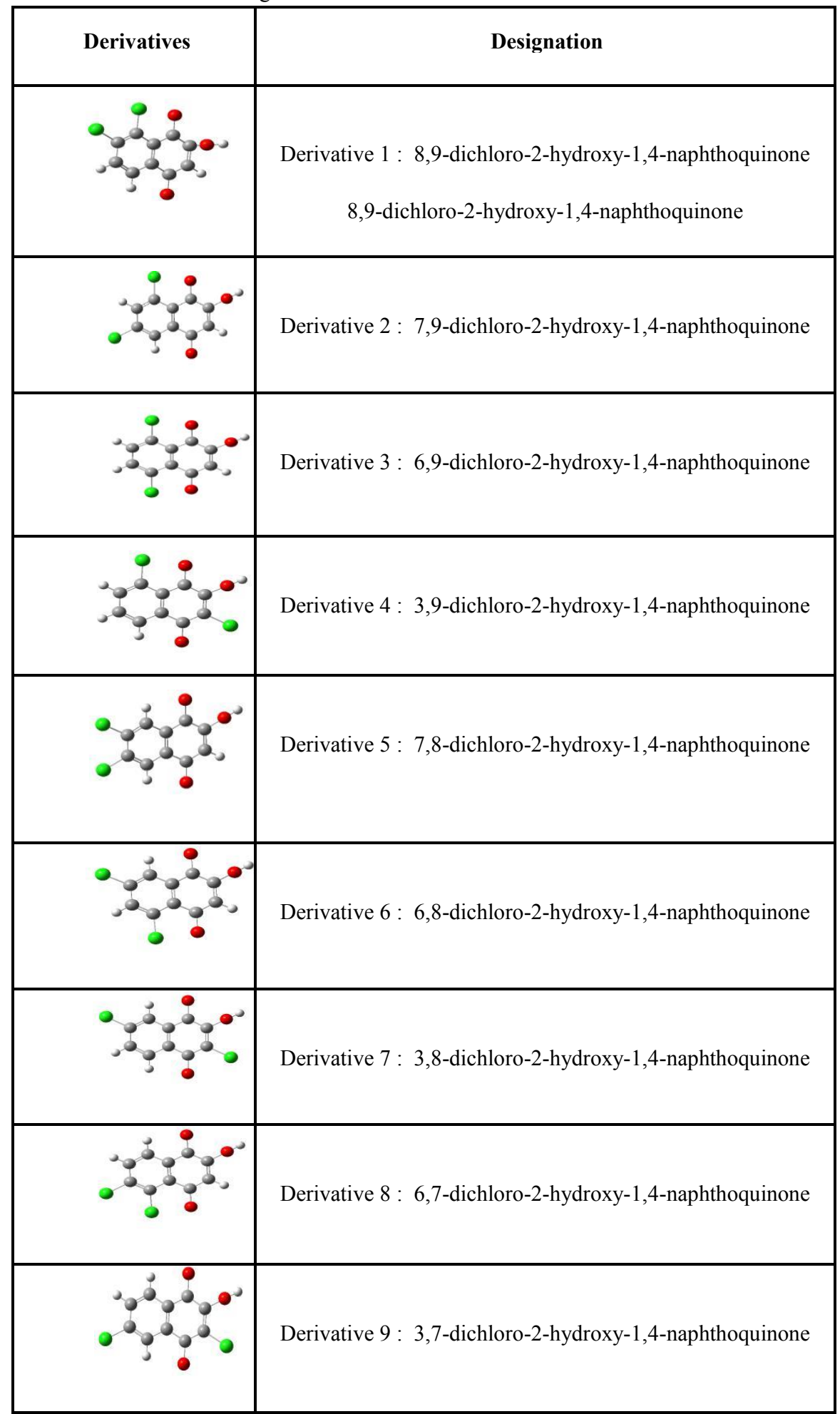




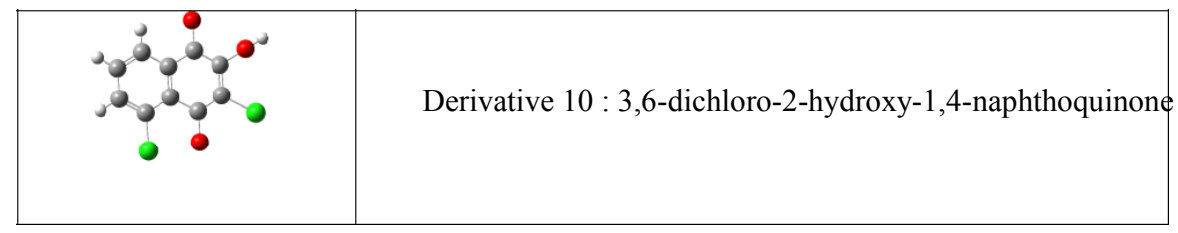

\section{Results and discussion}

Based on our calculations we have clarified geometrical stability of different 2-hydroxy-1,4-naphthoquinone and ten di-chlorinated derivatives in the neutral, cationic and anionic states. For the optimized structures the slight change in orientation of 2-hydroxy-1,4-naphthoquinone molecule results from planar geometrical configuration. The calculated quantum parameters of Lawsone are gathered in the following Table 2:

Table 2. Calculated properties of Lawsone molecule using B3LYP/6-311G** basis set.

\begin{tabular}{|c|c|c|c|c|c|c|c|c|}
\hline & $\begin{array}{c}E_{\text {HOMO }} \\
(\mathrm{eV})\end{array}$ & $\begin{array}{c}E_{\text {LUMO }} \\
(\mathrm{eV})\end{array}$ & $\begin{array}{c}E_{\text {gap }} \\
(\mathrm{eV})\end{array}$ & $\begin{array}{c}I \\
(\mathrm{eV})\end{array}$ & $\begin{array}{c}A \\
(\mathrm{eV})\end{array}$ & $\begin{array}{c}\chi \\
(\mathrm{eV})\end{array}$ & $\begin{array}{c}\eta \\
(\mathrm{eV})\end{array}$ & $\begin{array}{c}\Delta \mathrm{N}_{\mathrm{Fe}-} \\
\text { inh }\end{array}$ \\
\hline $\begin{array}{l}\text { Lawsone } \\
\text { Molecule }\end{array}$ & -0.2697 & -0.11691 & 0.15279 & -610.16 & -610.57 & 0.12 & 0.2 & 2.53 \\
\hline
\end{tabular}

On the other hand the analysis of HOMO and LUMO orbitals (Fig.1 a \& b) shows that the electronic structure of the molecule is dominated by the $\pi$ orbital. To examine the inhibitory properties of Lawsone iron corrosion, we first examined its orbital boundaries FMO depicted in Fig.1. These frontiers orbitals are used to predict inhibition efficiency of the molecule by considering the simplest transfer of electrons where the softness, local calculated properties, has the highest value. We have found that derivatives 3, 4, 7, 9 and 10 indicated in Table 1, have a higher HOMO- energy than that of Lawsone. This increase in HOMO energy is likely to facilitate adsorption on the metal surface, and hence inhibition, by influencing the transfer process through the adsorbed layer.

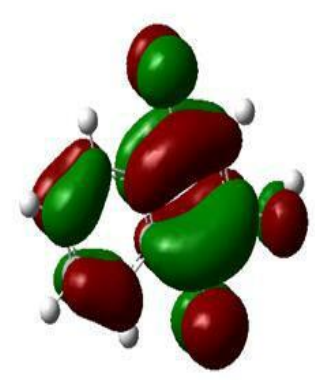

(a)

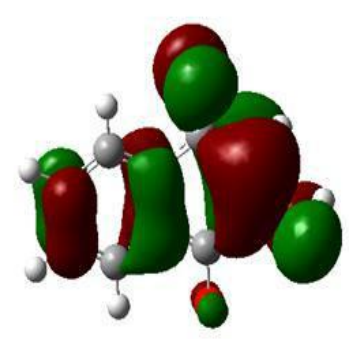

(b)

Fig. 1. (a) HOMO and (b) LUMO molecular orbital frontier of Lawsone at B3LYP/6-311G** optimized structure. 


\section{Conclusion}

The present study aims to clarify the correlation between the electronic properties of 2-hydroxy-1,4-naphthoquinone and ten di-chlorinated derivatives in the neutral, cationic and anionic states and the inhibition efficiency in contact with an iron atom. Our preliminary results indicated that the effect of strong inhibition power is attributed to the large fraction $\Delta \mathrm{N}$ of charge transfer, the relative position of HOMO energy, and the relatively low value of the gap energy gap as compared to that of the Lawsone molecule.

\section{References}

1. M. F. Iozzi, M. Cossi, R. Improta, N. Rega, V. Barone, J. Chem. Phys. 124, 184103 (2006)

2. K. Jug, T. Bredow, J. Comput. Chem. 25, 1551 (2004)

3. W. Kohn, L. J. Sham, Phys. Rev. 140, A1133 (1965)

4. P. Hohenberg, W. Kohn, Phys. Rev. 136, B 864 (1964)

5. R. Belkada, T. Igarashi, S. Ogata, Comput. Mater. Sci. 30, 195 (2004)

6. M. J. Frisch, Gaussian, Inc, Wallingford CT, Gaussian 09, Revision C.01 (2010) 